\begin{tabular}{|c|c|}
\hline Title & Finite element solution of anisotropic waveguides with arbitrary tensor permittivity \\
\hline Author(s) & Koshiba, M.; Hay ata, K.; Suzuki, M. \\
\hline Citation & Journal of Lightwave Technology, 4(2), 121-126 \\
\hline Issue Date & 1986-02 \\
\hline DOC URL & http:/hdl .handle.net/2115/6085 \\
\hline Rights & $\begin{array}{l}\text { O1986 IEEE. Personal use of this material is permitted. However, permission to reprint/republish this material for } \\
\text { advertising or promotional purposes or for creating new collective works for resale or redistribution to servers or lists, } \\
\text { or to reuse any copyrighted component of this work in other works must be obtained from the IEEE. } \\
\text { IEEE, Journal of Lightwave Technol ogy, } 4(2), 1986, \text { p121-126 }\end{array}$ \\
\hline Tyре & article \\
\hline File Information & JLT4_2.pdf \\
\hline
\end{tabular}

Instructions for use 


\title{
Finite-Element Solution of Anisotropic Waveguides with Arbitrary Tensor Permittivity
}

\author{
MASANORI KOSHIBA, SENIOR MEMBER, IEEE, KAZUYA HAYATA, AND \\ MICHIO SUZUKI, SENIOR MEMBER, IEEE
}

\begin{abstract}
An improved vector finite-element method has been used for the solution of general anisotropic waveguide problems. This method is formulated in terms of all three components of the magnetic field and is valid for arbitrary tensor permittivity. In the improved finite-element analysis, the spurious nonphysical solutions do not appear when the effective refractive index is larger than 1 . Therefore, this method is very useful for the analysis of the surface-wave modes of optical waveguides. To show the validity and usefulness of the improved finite-element method, computed results are illustrated for anisotropic rectangular waveguides with optic axis in any orientation and gyrotropic rectangular waveguides.
\end{abstract}

\section{INTRODUCTION}

A VECTOR finite-element method in an axial-component $\left(E_{z}-H_{z}\right)$ formulation [1]-[7] or in a three-component (the magnetic field $H$ ) formulation [8], [9] is widely used for the analysis of optical waveguides [2][4], [6], [7], [9]. The most serious difficulty in using the vector finite-element analysis is the appearance of the spurious nonphysical solutions [1], [5]-[9]. Recently, an improved finite-element method has been formulated in terms of all three components of $H$ [10]-[15]. In this improved $H$-field formulation, the spurious solutions do not appear when the effective refractive index $\beta / k_{0}$ is larger than 1 [14], [15], where $k_{0}$ is the wavenumber of free space and $\beta$ is the phase constant in the direction ( $z$ axis) of propagation. Therefore, this method is very useful for the analysis of the surface-wave modes of optical waveguides which correspond to the solutions in the region $\beta$ / $k_{0} \geq 1$. In addition, the $H$-field formulation is valid for general anisotropic problems. However, in [10]-[15], an isotropic dielectric waveguide is mainly studied and the application of the $H$-field formulation is restricted to an anisotropic dielectric waveguide composed of a uniaxial material whose optic axis lies in the plane ( $x y$-plane) perpendicular to the $z$ axis [10], [13], [15].

In this paper, to show the validity and usefulness of the improved $\mathrm{H}$-field formulation for a variety of anisotropic waveguides, computed results are illustrated for anisotropic rectangular waveguides with optic axis in any orientation and gyrotropic rectangular waveguides.

Manuscript received March 18, 1985; revised June 1, 1985

The authors are with the Department of Electronic Engineering, Hokkaido University, Sapporo, 060 Japan.

IEEE Log Number 8406199.

\section{Finite-Element Method}

A variational expression with the divergence-free constraint $(\nabla \cdot H=0)$ which is imposed in a least-square sense is known to be [10]-[16]

$$
\begin{aligned}
\tilde{F}= & \iint_{\Omega}(\nabla \times H)^{*} \cdot\left([K]^{-1} \nabla \times H\right) d \Omega \\
& -k_{0}^{2} \iint_{\Omega} H^{*} \cdot H d \Omega
\end{aligned}
$$

$$
+\iint_{\Omega}(\nabla \cdot H)^{*}(\nabla \cdot H) d \Omega
$$

where $\Omega$ represents the cross section of a waveguide, the asterisk denotes a complex conjugate, and the relative permittivity tensor $[K]$ is given by

$$
[K]=\left[\begin{array}{lll}
K_{x x} & K_{x y} & K_{z x}^{*} \\
K_{x y}^{*} & K_{y y} & K_{y z} \\
K_{z x} & K_{y z}^{*} & K_{z z}
\end{array}\right] .
$$

Here [ $\cdot]$ denotes a matrix. As we consider loss-free materials, $[K]$ is Hermitian. In anisotropic dielectric cases, all elements of $[K]$ are real so that $[K]$ is a real symmetric matrix [17]. In gyrotropic cases, diagonal elements of $[K]$ are real, whereas off-diagonal ones in general are complex [17]. The former corresponds to materials with crystalline anisotropy or electrooptic cases, while the latter corresponds to the magnetooptic case [17].

Dividing the cross section $\Omega$ of the waveguide into a number of second-order triangular elements in Fig. 1, the magnetic fields $H_{x}, H_{y}$, and $H_{z}$ within each element are defined in terms of the magnetic fields at the corner and midside nodal points:

$$
\begin{aligned}
& H_{x}=\{N\}^{T}\left\{H_{x}\right\}_{e} \exp (-j \beta z) \\
& H_{y}=\{N\}^{T}\left\{H_{y}\right\}_{e} \exp (-j \beta z) \\
& H_{z}=j\{N\}^{T}\left\{H_{z}\right\}_{e} \exp (-j \beta z)
\end{aligned}
$$

where $\left\{H_{x}\right\}_{e},\left\{H_{y}\right\}_{e}$, and $\left\{H_{z}\right\}_{e}$ are magnetic field vectors corresponding to the nodal points within each element, $\{N\}$ is the shape function vector [10], [15], and $T,\{\cdot\}$, and $\{\cdot\}^{T}$ denote a transpose, a column vector, and a row 


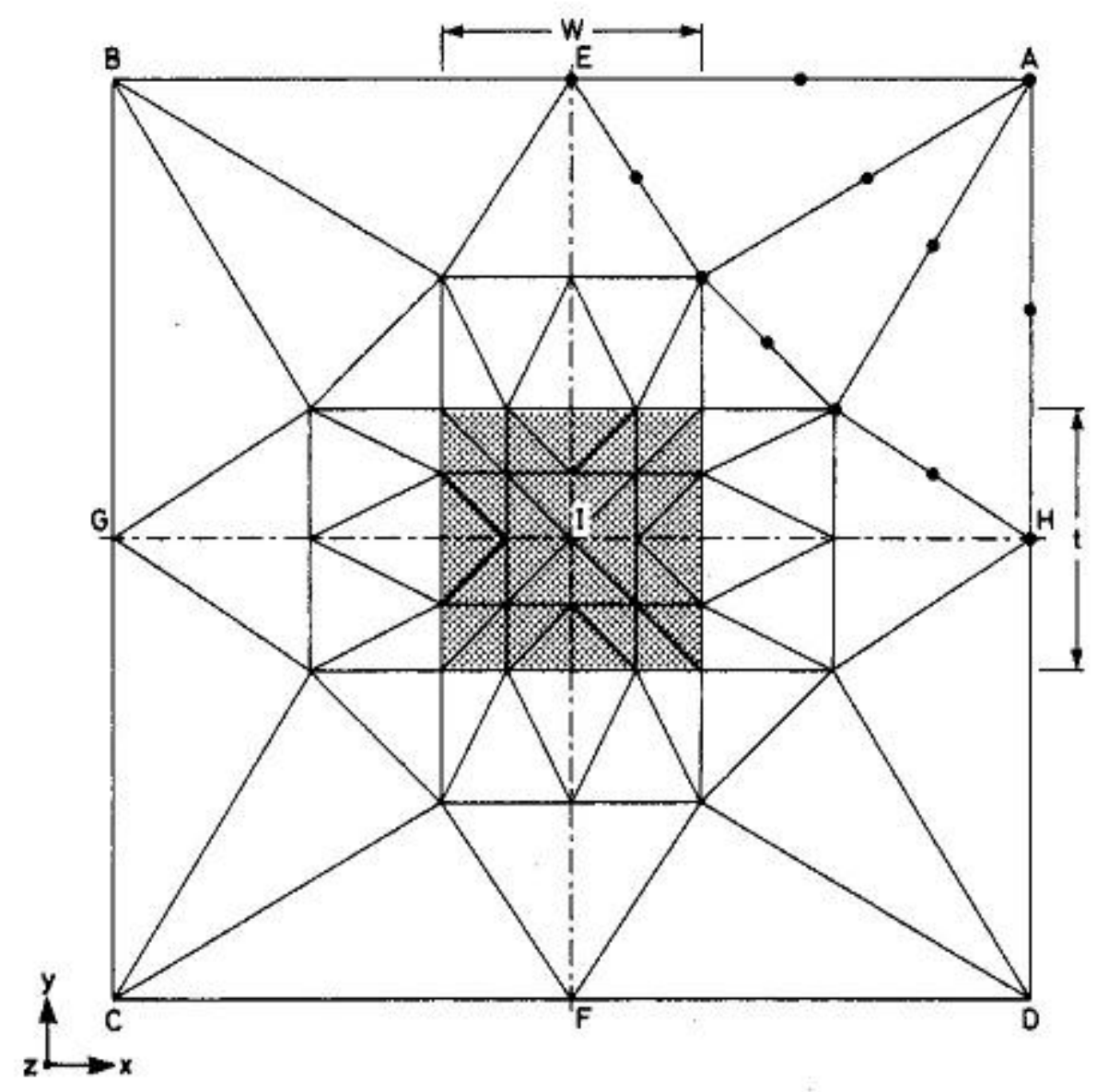

Fig. 1. Finite-element division of an anisotropic rectangular waveguide or a gyrotropic rectangular waveguide.

vector, respectively. Variation of (1) with respect to the nodal variables leads to the following eigenvalue problem:

$$
([S]+[U])\{H\}-k_{0}^{2}[T]\{H\}=\{0\}
$$

where $\{H\}$ is the nodal magnetic field vector, $\{0\}$ is a null vector, $[S],[T]$, and $[U]$ are the matrices related to the first, second, and third terms on the right-hand side of (1), respectively, and $[T]$ is a real symmetric matrix. For loss-free materials, $[U]$ is a real symmetric matrix and $[S]$ is a complex Hermitian matrix. When $\operatorname{Im}\left(K_{x y}\right)=\operatorname{Re}\left(K_{y z}\right)$ $=\operatorname{Re}\left(K_{z x}\right)=0,[S]$ becomes a real symmetric matrix [8], [13].

If the divergence-free constraint is neglected, the following eigenvalue equation is derived [8], [9], [18]:

$$
[S]\{H\}-k_{0}^{2}[T]\{H\}=\{0\} .
$$

In the earlier finite-element analysis using (7), the spurious solutions appear and these solutions are scattered all over the propagation diagram [8]-[16], [18].

In the improved finite-element analysis using (6), the appearance of the spurious solutions is limited to the region $\beta / k_{0}<1$ [14], [15]. They do not appear when the effective refractive index $\beta / k_{0}$ is larger than 1 . Therefore, (6) is very useful for the analysis of the surface-wave modes of optical waveguides which correspond to the solutions in the region $\beta / k_{0} \geq 1$.

\section{Computed Results}

In this section, we present the computed results for anisotropic rectangular waveguides and gyrotropic rectangular waveguides. The guided modes are designated as $E_{p q}^{x}$ or $E_{p q}^{y}$ [19]. The main field components of the $E_{p q}^{x}$ modes are $E_{x}$ and $H_{y}$, while those of the $E_{p q}^{y}$ modes are $E_{y}$ and $H_{x}$ [19]. A typical division of these waveguides into second-order triangular elements is shown in Fig. 1,

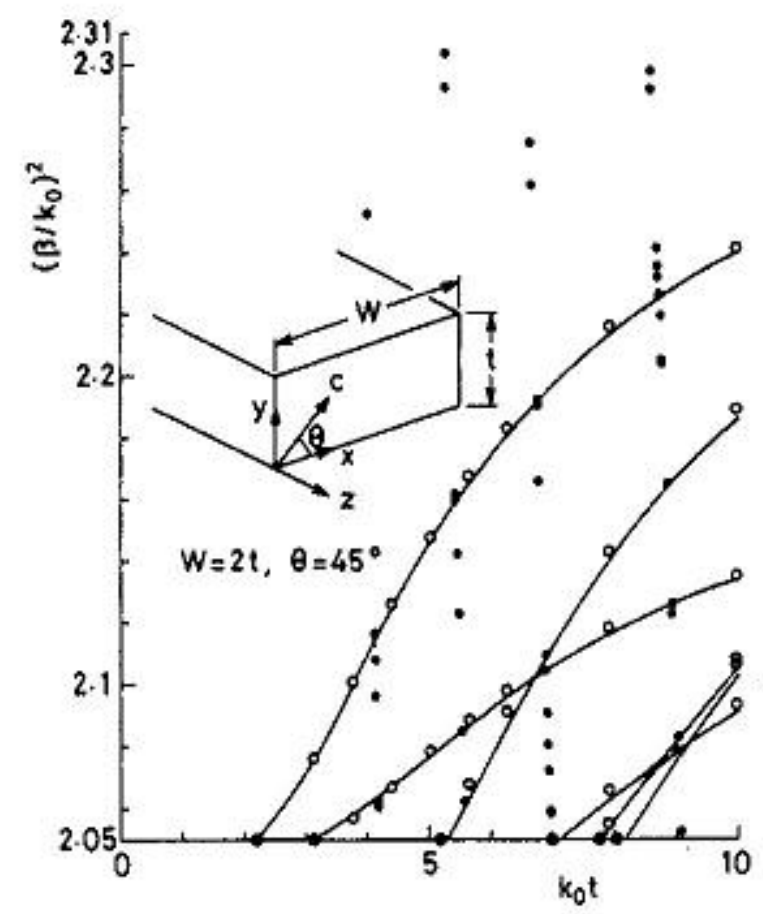

Fig. 2. Dispersion characteristics of an anisotropic rectangular waveguide. Solid lines, circles, and dots represent the solutions of (6), the results of the variational method [20], and the solutions of (7), respec. tively.

where the boundaries $A B, B C, C D$, and $D A$ are assumed to be perfect electric conductors [15]. The convergence of the solution is checked by moving these conductors gradually away from a core region.

\section{A. Anisotropic Rectangular Waveguides}

We consider an anisotropic rectangular waveguide composed of a uniaxial material surrounded by an iso tropic material of refractive index $\sqrt{2.05}$, where the ordinary and extraordinary refractive indices of a rectangular core are $\sqrt{2.31}$ and $\sqrt{2.19}$, respectively.

Fig. 2 shows the dispersion characteristics of the anisotropic rectangular waveguide with $W=2 t$ whose optic axis $c$ lies in the $x y$-plane at an angle $\theta=45^{\circ}$ from the $x$ axis, where the whole region $A B C D$ in Fig. 1 should be divided into elements because of the lack of symmetry of the field. The solid lines in Fig. 2 represent the solutions of the improved finite-element program in (6). Compari son of our results with the results of the variational method [20] indicated by circles shows good agreement. This fact demonstrates the reliability of the present method. The dots in Fig. 2 represent the solutions of the earlier finite element program in (7). It is found that when (7) is used numerous spurious solutions appear. The guided modes of the waveguide in Fig. 2 can not be designated as $E_{p \bar{q}}^{x}$ or $E_{p q}^{y}$ because the main magnetic field component of these guided modes is not $H_{x}$ or $H_{y}$ [20].

Figs. 3 and 4 show the dispersion characteristics of the anisotropic square waveguides $(W=t)$ whose optic axis lies in the $x y$-plane at an angle $\theta$ from the $x$ axis. The waveguide with $\theta=0^{\circ}$ in Fig. 3 has two planes of sym metry. Therefore, for the waveguide in Fig. 3, the region $A E I H$ in Fig. 1 is divided into elements. The $E_{11}^{y}$ and the $E_{11}^{x}$ modes in Fig. 3 are the fundamental and the first higher-order modes, respectively. In an isotropic square waveguide, the $E_{11}^{y}$ and the $E_{11}^{x}$ modes are degenerate [19]. The propagation diagram of the waveguide with $\theta$ $=45^{\circ}$ in Fig. 4 is very similar to that in Fig. 3. However the eigenvectors for the guided modes of the waveguide 


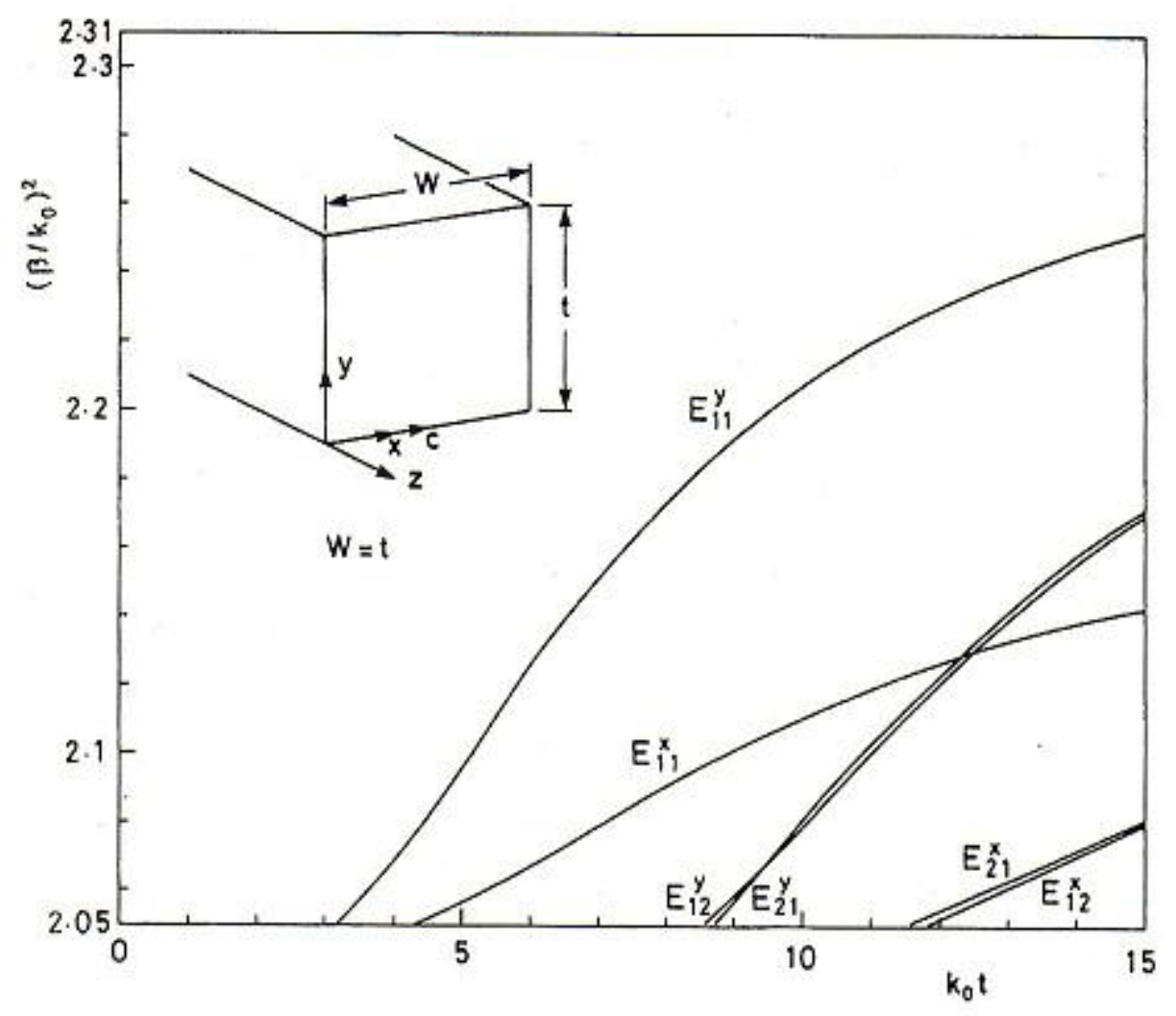

Fig. 3. Dispersion characteristics of an anisotropic square waveguide with the optic axis parallel to the $x$ axis.

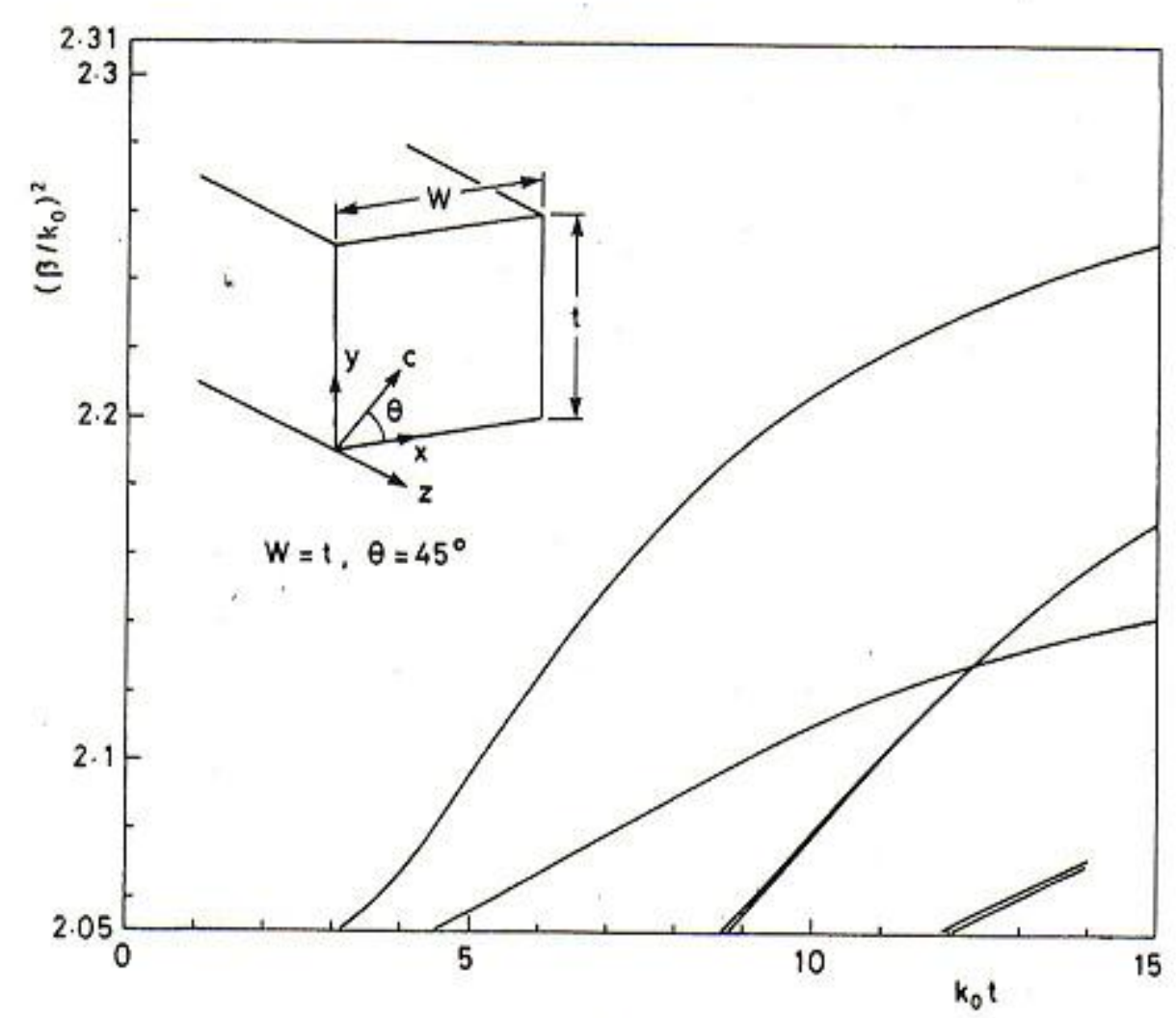

Fig. 4. Dispersion characteristics of an anisotropic square waveguide whose optic axis lies in the $x y$-plane at an angle $\theta=45^{\circ}$ from the $x$ axis.

in Fig. 4 are different from those in Fig. 3. For the fundamental and the first higher-order modes, the variations of the transverse components of the magnetic field vector in a core-region with $\theta$ are shown in Figs. 5 and 6 , respectively, where $\beta t=14$. The directions of the magnetic field for the fundamental and the first higher-order modes are almost parallel and almost normal to the optic axis, respectively. Therefore, the guided modes of the waveguide in Fig. 4 can not be designated as $E_{p q}^{x}$ or $E_{p q}^{y}$.

Figs. 7 and 8 show the dispersion characteristics of the anisotropic square waveguides whose optic axis does not lie in the $x y$-plane. The optic axis lies in the $y z$-plane at an angle $\theta$ from the $y$ axis. The waveguide with $\theta=90^{\circ}$ in Fig. 7 has two planes of symmetry, and therefore the region $A E I H$ in Fig. 1 is divided into elements. On the other hand, the waveguide with $\theta=45^{\circ}$ in Fig. 8 has one plane of symmetry, and therefore the region $A E F D$ in Fig. 1 is divided into elements. In the waveguide in Fig. 7, the $E_{11}^{x}$ and the $E_{11}^{y}$ modes are degenerate. When $\theta \neq$ $90^{\circ}$, these modes are no longer degenerate and the $E_{11}^{x}$

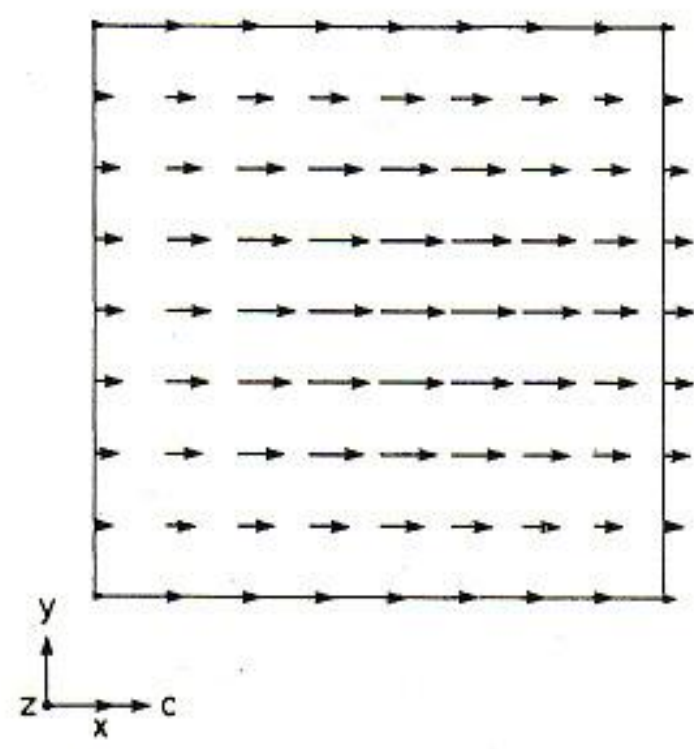

(a)

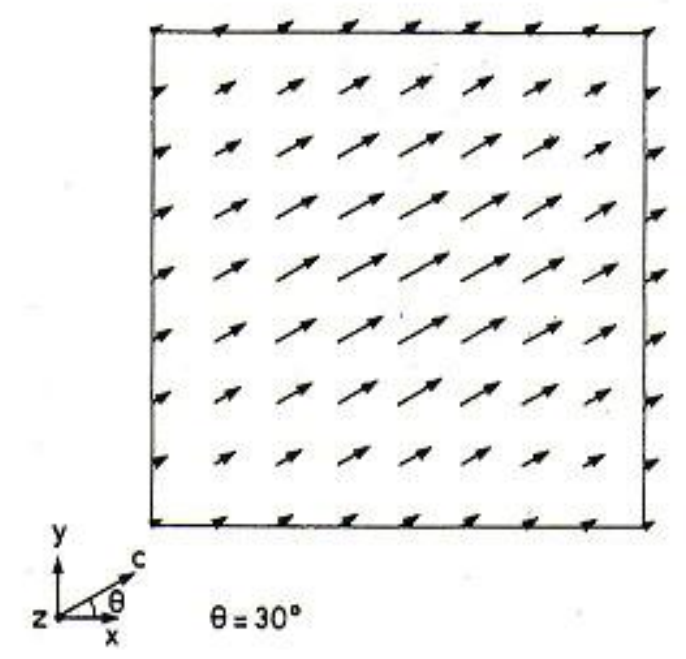

(c)

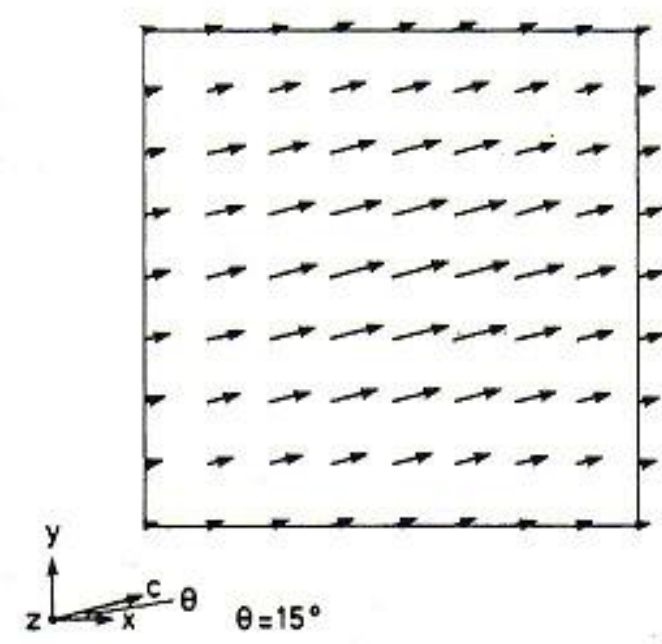

(b)

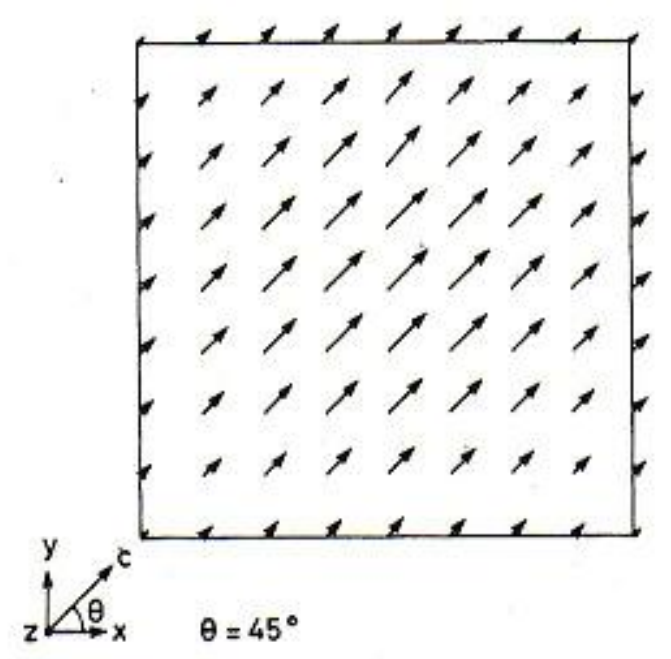

(d)
Fig. 5. Magnetic field vector for the fundamental mode of anisotropic square waveguides whose optic axis lies in the $x y$-plane at an angle $\theta$ from the $x$ axis. (a) $\theta=0^{\circ}$. (b) $\theta=15^{\circ}$. (c) $\theta=30^{\circ}$. (d) $\theta=45^{\circ}$.

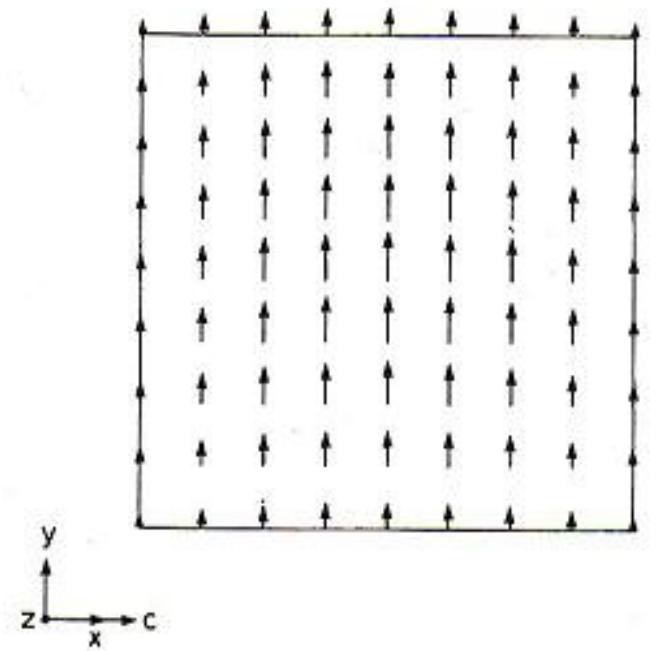

(a)

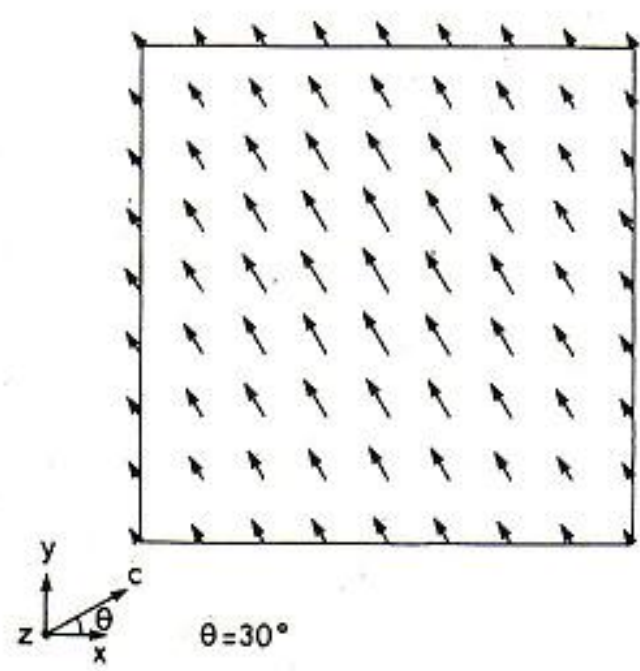

(c)

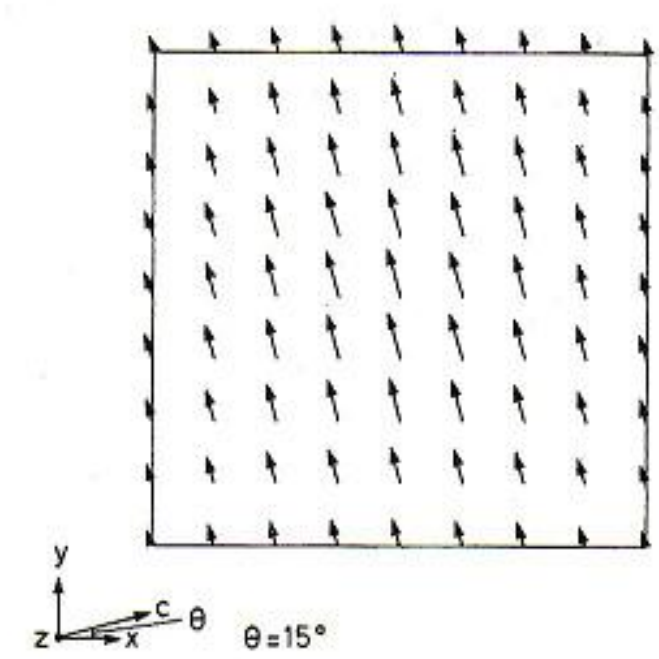

(b)

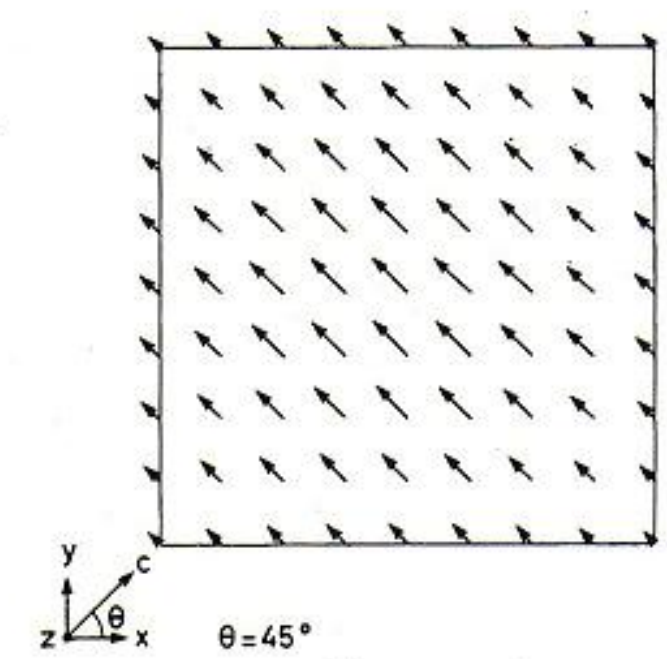

(d)
Fig. 6. Magnetic field vector for the first higher-order mode of anisotropic square waveguides whose optic axis lies in the $x y$-plane at an angle $\theta$ from the $x$ axis. (a) $\theta=0^{\circ}$. (b) $\theta=15^{\circ}$. (c) $\theta=30^{\circ}$. (d) $\theta=45^{\circ}$.

mode is the fundamental mode. The effect of the orientation of the optic axis is much stronger on the $E_{11}^{y}$ mode than the $E_{11}^{x}$ mode. 


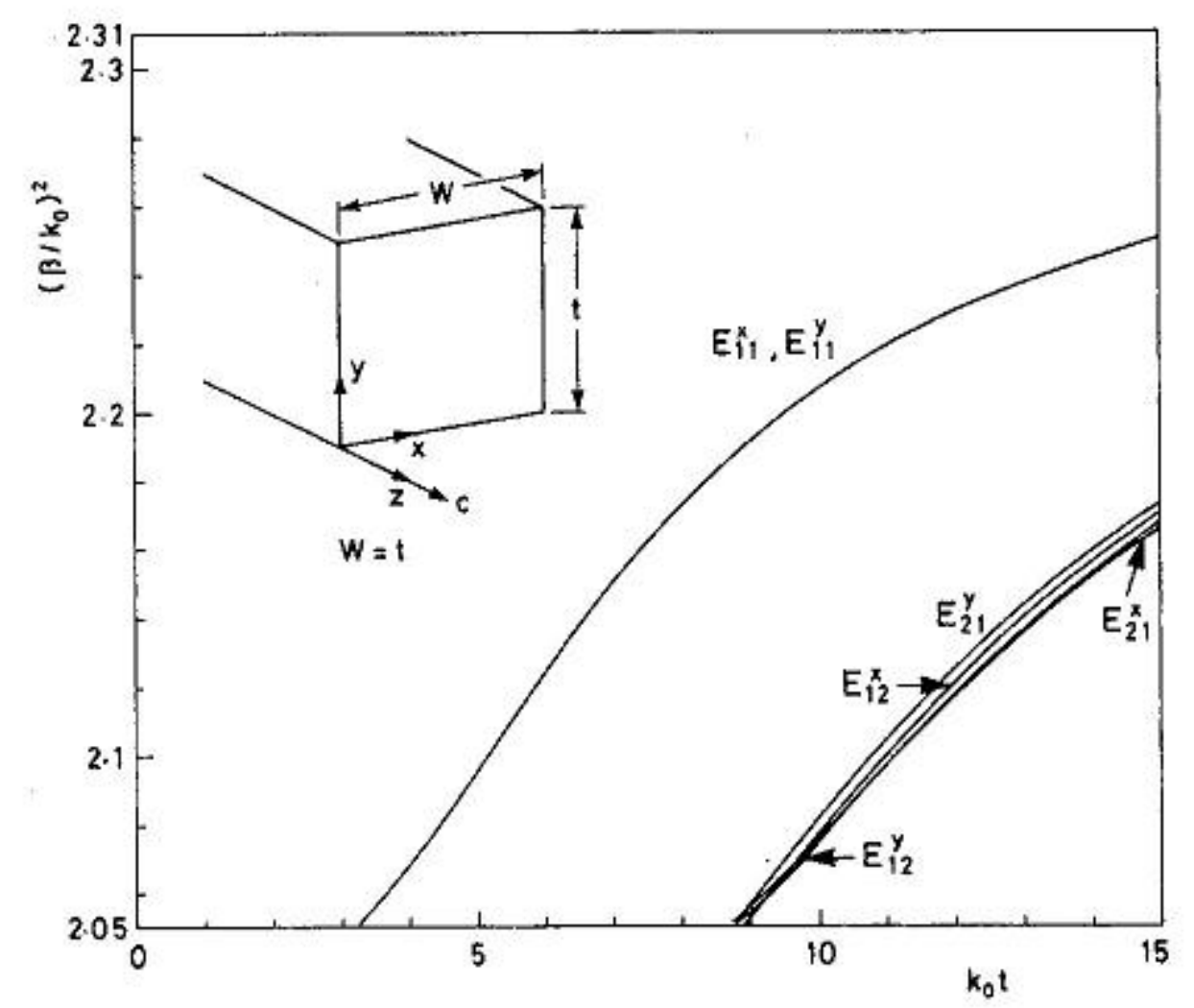

Fig. 7. Dispersion characteristics of an anisotropic square waveguide with the optic axis parallel to the $z$ axis.

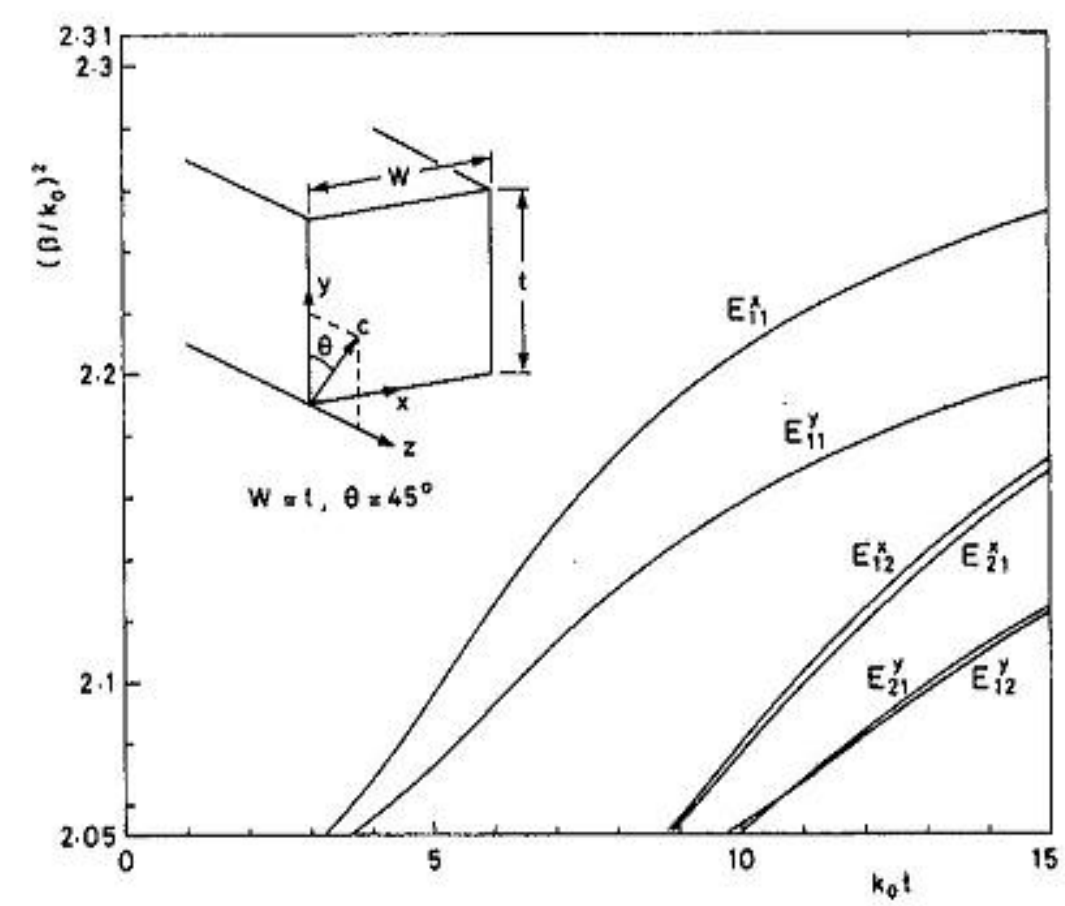

Fig. 8. Dispersion characteristics of an anisotropic square waveguide whose optic axis lies in the $y z$-plane at an angle $\theta=45^{\circ}$ from the $y$ axis.

\section{B. Gyrotropic Rectangular Waveguides}

We consider a gyrotropic rectangular waveguide surrounded by air, where the relative permittivity tensor of the waveguide is given by

$$
[K]=\left[\begin{array}{ccc}
K_{x x} & 0 & 0 \\
0 & K_{y y} & K_{y z} \\
0 & K_{y z}^{*} & K_{z z}
\end{array}\right] .
$$

Here $K_{y y}=K_{z z}=3$ and $K_{y z}$ is pure imaginary. This waveguide has one plane of symmetry, and therefore the region $A E F D$ in Fig. 1 is divided into elements.

Fig. 9 shows the dispersion characteristics of the gyrotropic square waveguides $(W=t)$. When $K_{x x}=3$ and $K_{y z}=0$, the $E_{11}^{x}$ and the $E_{11}^{y}$ modes are degenerate. In other cases, these modes are no longer degenerate. For $K_{x x}=2\left(K_{x x}<K_{y y}=K_{z z}\right)$ or $K_{x x}=4\left(K_{x x}>K_{y y}=K_{z z}\right)$, the fundamental mode is the $E_{11}^{y}$ or the $E_{11}^{x}$ mode, respectively. The effect of $K_{x x}$ is much stronger on the $E_{11}^{x}$ mode than the $E_{11}^{y}$ mode. In the $E_{11}^{x}$ mode, the effective refractive indices for $K_{y z} \neq 0$ is larger than those for $K_{y z}=0$.

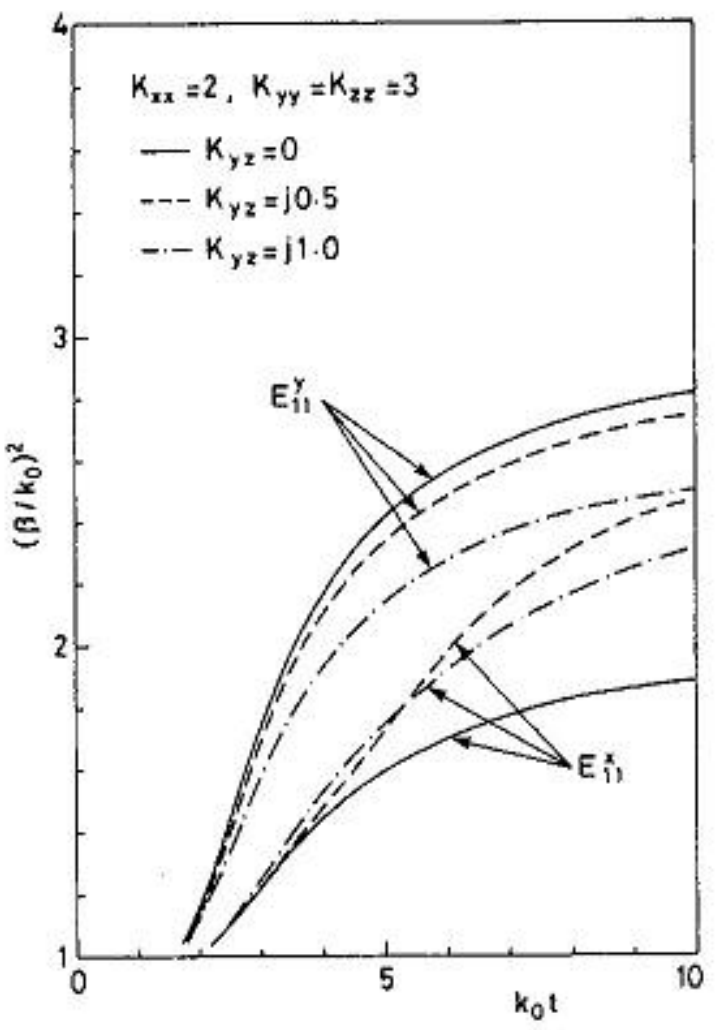

(a)

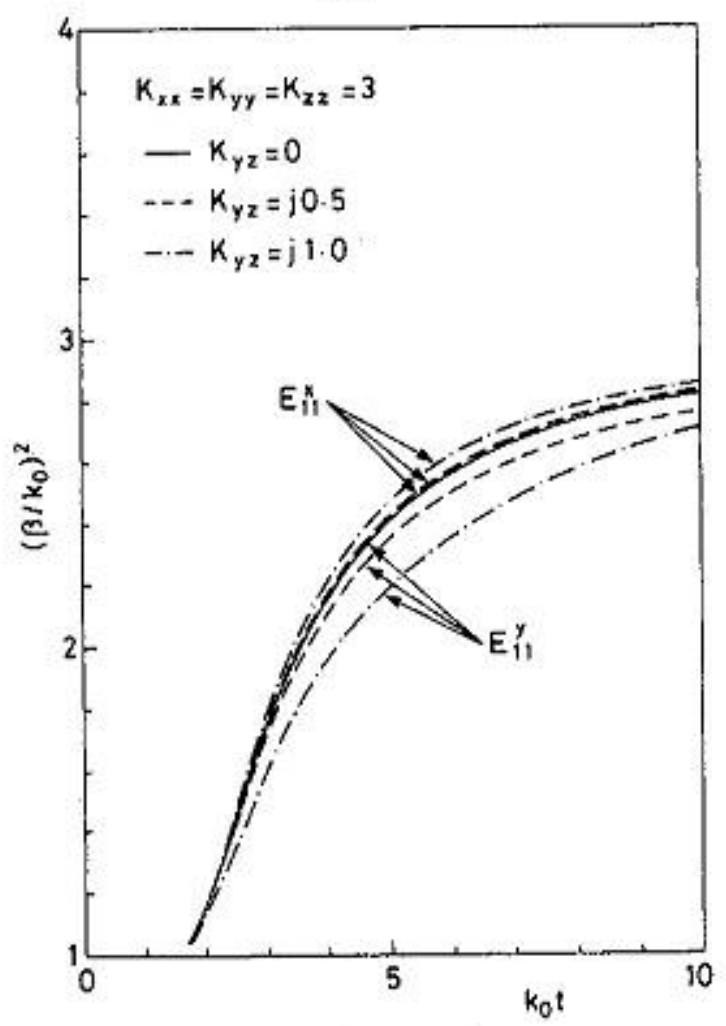

(b)

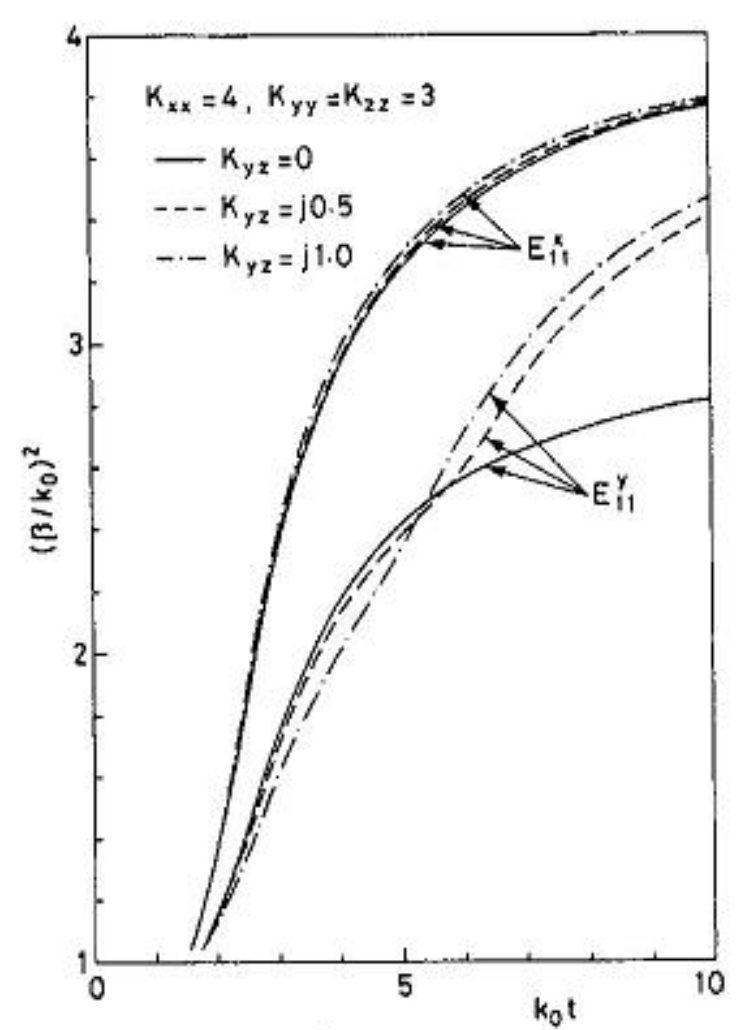

(c)

Fig. 9. Dispersion characteristics of gyrotropic square waveguides. (a) $K_{x x}$ =2. (b) $K_{x x}=3$. (c) $K_{x x}=4$.

In the $E_{11}^{y}$ mode, on the other hand, the effective refractive indices for $K_{y z} \neq 0$ is smaller than those for $K_{y z}=0$, except the higher frequency range for $K_{x x}=4$.

For the gyrotropic square waveguide with the off-di- 
agonal element $K_{z x}\left(K_{x y}=K_{y z}=0\right)$, the region $A B G H$ in Fig. 1 is divided into elements. The dispersion curves for the $E_{11}^{x}$ or the $E_{11}^{y}$ mode of this waveguide are the same as those for the $E_{11}^{y}$ or the $E_{11}^{x}$ mode of the waveguide in Fig. 9, respectively, where $K_{x x}, K_{y y}$, and $K_{y z}$ in Fig. 9 should be replaced by $K_{y y}, K_{x x}$, and $K_{z x}$, respectively.

For the gyrotropic square waveguide with the off-diagonal element $K_{x y}\left(K_{y z}=K_{z x}=0\right)$, the region $A B C D$ in Fig. 1 is divided into elements. The guided modes of this waveguide may not be designated as $E_{p q}^{x}$ or $E_{p q}^{y}$ in the same manner as the cases of Figs. 2 and 4 .

In Figs. 3, 4, and 7-9, the spurious solutions do not appear because $\beta / k_{0} \geq 1$.

\section{CONCLUSIONS}

Here we have shown the capability of the improved finite-element method in the three-component magnetic field formulation for solving general anisotropic waveguide problems. In this approach, the spurious solutions do not appear when the effective refractive index is larger than 1 . Therefore, this method is very useful for the analysis of the surface-wave modes of optical waveguides. The validity of the method was confirmed by comparing numerical results for the anisotropic rectangular waveguide with the results of the variational method. The numerical results for the anisotropic square waveguides and the gyrotropic square waveguides were presented and the propagation characteristics of each waveguide was discussed.

\section{REFERENCES}

[1] P. Daly, "Hybrid-mode analysis of microstrip by finite-element methods," IEEE Trans. Microwave Theory Technol., vol. MTT-19, pp. 19-25, Jan. 1971.

[2] C. Yeh, S. B. Dong, and W. Oliver, "Arbitrarily shaped inhomogeneous optical fiber or integrated optical waveguides," $J$. Appl. Phys., vol. 46, pp. 2125-2129, May 1975.

[3] P. Vandenbulcke and P. E. Lagasse, "Eigenmode analysis of anisotropic optical fibres or integrated optical waveguides, " Electron. Lett., vol. 12, pp. 120-122, Mar. 1977.

[4] C. Yeh, K. Ha, S. B. Dong, and W. P. Brown, "'Single-mode optical waveguides," Appl. Opt., vol. 18, pp. 1490-1504, May 1979.

[5] M. Ikeuchi, H. Sawami, and H. Niki, "Analysis of open-type dielectric waveguides by the finite-element iterative method," IEEE Trans. Microwave Theory Technol., vol. MTT-29, pp. 234-239, Mar. 1981.

[6] N. Mabaya, P. E. Lagasse, and P. Vandenbulcke, "Finite element analysis of optical waveguides," IEEE Trans. Microwave Theory Technol., vol. MTT-29, pp. 600-605, June 1981.

[7] K. Oyamada and T. Okoshi, "Two-dimensional finite-element calculation of propagation characteristics of axially nonsymmetrical optical fibers,"' Radio Sci., vol. 17, pp. 109-116, Jan.-Feb. 1982.

[8] A. Konrad, "High-order triangular finite elements for electromagnetic waves in anisotropic media,"' IEEE Trans. Microwave Theory Technol., vol. MTT-25, pp. 353-360, May 1977.

[9] B. M. A. Rahman and J. B. Davies, "Finite-element analysis of optical and microwave waveguide problems," IEEE Trans. Microwave Theory Technol., vol. MTT-32, pp. 20-28, Jan. 1984.

[10] M. Koshiba, K. Hayata, and M. Suzuki, "Vectorial finite-element formulation without spurious modes for dielectric waveguides," Trans. Inst. Electron. Commun. Eng. Japan, vol. E67, pp. 191-196, Apr. 1984.

[11] M. Koshiba, K. Hayata, and M. Suzuki, "Vectorial finite-element method without spurious solutions for dielectric waveguide problems,"'Electron. Lett., vol. 20, pp. 409-410, May 1984.

[12] B. M. A. Rahman and J. B. Davies, "Penalty function improvement of waveguide solution by finite elements," IEEE Trans. Microwave Theory Technol., vol. MTT-32, pp. 922-928, Aug. 1984.

[13] B. M. A Rahman and J. B. Davies, "Finite-element solution of integrated optical waveguides," J. Lightwave Technol., vol. LT-2, pp. 682-688, Oct. 1984.

[14] M. Koshiba, K. Hayata, and M. Suzuki, "'Study of spurious solutions of finite-element methods in the three-component magnetic field formulation for dielectric waveguide problems," Trans. Inst. Electron. Commun. Eng. Japan, vol. J67-B, pp. 1333-1338, Dec. 1984 (in Japanese).

[15] M. Koshiba, K. Hayata, and M. Suzuki, "Improved finite-element formulation in terms of the magnetic-field vector for dielectric waveguides," IEEE Trans. Microwave Theory Technol., vol. MTT-33, pp. 227-233, Mar. 1985.

[16] M. Hara, T. Wada, T. Fukasawa, and F. Kikuchi, "A three-dimensional analysis of RF electromagnetic fields by the finite element method,"' IEEE Trans. Magn., vol. MAG-19, pp. 2417-2420, Nov. 1983.

[17] S. Yamamoto, Y. Koyamada, and T. Makimoto, "Normal-mode analysis of anisotropic and gyrotropic thin-film waveguides for integrated optics,"' J. Appl. Phys., vol. 43, pp. 5090-5097, Dec. 1972.

[18] J. B. Davies, F. A. Fernandez, and G. Y. Philippou, "Finite element analysis of all modes in cavities with circular symmetry," IEEE Trans. Microwave Theory Technol., vol. MTT-30, pp. 1975-1980, Nov. 1982.

[19] E. A. J. Marcatili, "Dielectric rectangular waveguide and directional coupler for integrated optics," Bell Syst. Tech. J., vol. 48, pp. 20712102 , Sept. 1969.

[20] M. Ohtaka, "Analysis of the guided modes in the anisotropic dielectric rectangular waveguides," Trans. Inst. Electron. Commun. Eng. Japan, vol. J64-C, pp. 674-681, Oct. 1981 (in Japanese).

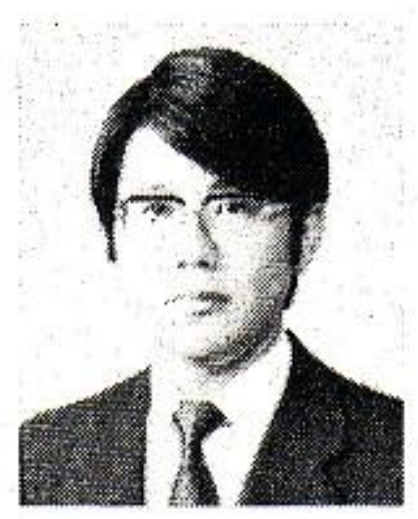

Masanori Koshiba (SM'84) was born in Sapporo, Japan, on November 23, 1948. He received the B.S., M.S., and Ph.D. degrees in electronic engineering from Hokkaido University, Sapporo, Japan, in 1971, 1973, and 1976, respectively.

In 1976, he joined the Department of Electronic Engineering, Kitami Institute of Technology, Kitami, Japan. Since 1979, he has been an Assistant Professor of Electronic Engineering at Hokkaido University. He has been engaged in research on surface acoustic waves, dielectric optical waveguides, and applications of finite-element and boundary-element methods to field problems.

Dr. Koshiba is a member of the Institute of Electronics and Communication Engineers of Japan, the Institute of Television Engineers of Japan, the Institute of Electrical Engineers of Japan, the Japan Society for Simulation Technology, and Japan Society for Computational Methods in Engineering.

$*$

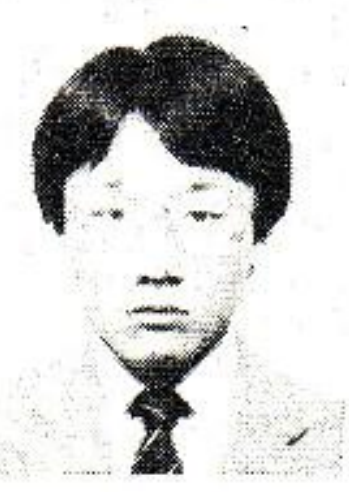

Kazuya Hayata was born in Kushiro, Japan, on December 1,1959 . He received the B.S. and M.S. degrees in electronic engineering from Hokkaido University, Sapporo, Japan, in 1982 and 1984, respectively.

Since 1984, he has been a Research Assistant of Electronic Engineering at Hokkaido University. He has been engaged in research on dielectric optical waveguides and surface acoustic waves.

Mr. Hayata is a member of the Institute of Electronics and Communication Engineers of Japan. 


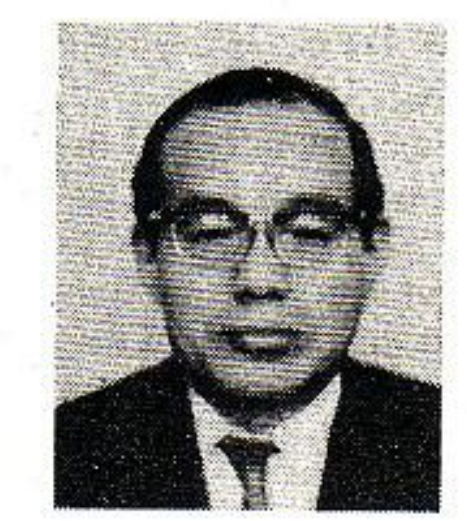

Michio Suzuki (SM'57) was born in Sapporo, Japan, on November 14, 1923. He received the B.S. and $\mathrm{Ph} . \mathrm{D}$. degrees in electrical engineering from Hokkaido University, Sapporo, Japan, in 1946 and 1960 , respectively.

From 1948 to 1962 , he was an Assistant Professor of Electrical Engineering at Hokkaido University. Since 1962, he has been a Professor of Electronic Engineering at Hokkaido University. From 1956 to 1957, he was a Research Associate at the Microwave Research Institute of Polytechnic Institute of Brooklyn, Brooklyn, NY.
Dr. Suzuki is a member of the Institute of Electronics and Communication Engineers of Japan, the Institute of Electrical Engineers of Japan, the Institute of Television Engineers of Japan, the Japan Society of Information and Communication Research, and the Japan Society for Simulation Technology. 